\title{
The Effect of 2-deoxy-D-Glucose on the Growth and Respiration of Coprinus lagopus
}

\author{
By D. MOORE \\ The Department of Botany, The University, Manchester, I3 \\ (Accepted for publication 19 February 1968) \\ SUMMARY
}

\begin{abstract}
The unnatural glucose analogue 2-deoxy-D-glucose was found to be fungistatic rather than fungicidal for a laboratory wild-type strain of the Basidiomycete Coprinus lagopus; the analogue was not used as a carbon + energy source. Extension-growth rate of the organism was profoundly affected by the presence of 2-deoxyglucose; but although this growth could be prevented completely the inhibition was readily annulled, either by the addition of an excess of the normal substrate or by transfer to normal media. The extent of the inhibition observed depended not only on the ratio of normal hexose to analogue, but also on the identity of the normal hexose in the medium. Coprinus lagopus was at least Ioo times more sensitive to the analogue when fructose was present than with glucose. This extreme sensitivity differential was also observed in the effect of 2-deoxyglucose on oxygen uptake by spore suspensions: inhibition of $\mathrm{O}_{2}$ uptake occurred, and was about Ioo times greater with fructose than with glucose.
\end{abstract}

\section{INTRODUCTION}

Woodward, Cramer \& Hudson (1953) reported that the unnatural glucose analogue 2-deoxy-D-glucose (deGlc) caused inhibition of growth of Saccharomyces. This was confirmed by Heredia, de la Fuente \& Sols (1964) who showed that growth and glycolysis were inhibited in Saccharomyces cerevisiae and $S$. fragilis. These workers showed that growth on glucose $+\operatorname{deGlc}(2 \%+0.1 \%)$ was only about $30 \%$ of that on normal hexoses, while virtually no growth was made on fructose + deGlc at the same concentrations. Incubation of organisms with fructose + deGlc lead to the death of over $95 \%$ of active organisms in about $24 \mathrm{hr}$.

The growth of Neurospora crassa was decreased on media containing equimolar concentrations of deGlc and glucose, though with limiting glucose the addition of analogue increased the final growth yield (Sols, Heredia \& Ruiz-Amil, 1960). Neurospora crassa can utilize deGlc as sole carbon + energy source although the growth is weak. On the other hand Aspergillus oryzae utilizes deGlc fairly readily, the growth rate being about one fifth of that on normal hexose (Sols et al. 1960). Barban \& Schulze (I96I) working with human cell cultures, showed that there was essentially no growth at equimolar concentrations of deGlc + glucose and that after about 3 days of exposure to this medium the cytopathogenic effects were irreversible, cells grown on fructose were 5 to Io times more sensitive to inhibition than those grown on glucose. The respiration of yeasts (Heredia et al. 1964) and human cells (Barban \& Schulze, 196r) was markedly inhibited by deGlc. In the case of yeasts the inhibition of respiration was not as great as the inhibition of growth and was not a direct cause of the latter. 
Enzymic studies have shown that deGlc is readily phosphorylated by hexokinase (ATP: D-hexose 6-phosphotransferase, E.C. no. 2.7.I.I.) (Heredia et al. 1964; Barban \& Schulze, I96I; Sols et al. 1960) without inhibiting glucose phosphorylation (Barban \& Schulze, 196I). However, the phosphorylation of fructose was actively inhibited by deGlc in HeLa cell extracts. Phosphohexoseisomerase (D-glucose-6-phosphate ketolisomerase, E.C. no. 5.3.I.9.) and glucose-6-phosphate dehydrogenase (D-glucose-6phosphate: NADP oxidoreductase, E.C. no. I.I.I. 49.) from HeLa cells were both inhibited by deGlc-6-phosphate (Barban \& Schulze, 196I). The available evidence indicates that the inhibitory effects of deGlc are conditional on its phosphorylation. Inhibition of the entry of normal sugars into the cell by accumulated deGlc phosphates and possibly inhibition of early enzymes of glycolysis and of the pentose phosphate cycle are probably sufficient to account for the effects seen on respiration. The lethal effect observed with yeasts (Heredia et al. 1964 ; Megnet, 1965) is probably accounted for by a trapping of uridine nucleotides by phosphorylated derivatives of deGlc, with a consequent interference with the synthesis of structural polysaccharides (Heredia et al. 1964). A deGlc-resistant mutant of yeast (Heredia \& Sols, 1964) and a resistant sub-line of HeLa cells (Barban, I962) have been described. In both cases growth (40\% to $50 \%$ of normal) was obtained with equimolar concentrations of normal hexose + deGlc-conditions under which the parental strains showed no growth. The evidence indicated that resistance in each case was due to an abnormally high phosphatase activity which decreased the intracellular deGlc phosphate concentration. The general effects of deGlc in a wide range of systems were reviewed by Webb (1966).

The present paper describes work on the effects of deGlc on the Basidiomycete Coprinus lagopus. The particular interest of this organims lies in the observation that it is naturally highly resistant to the analogue in the presence of glucose, but very sensitive in the presence of fructose.

\section{METHODS}

The organism used was a strain of Coprinus lagopus, isolation number $\mathrm{BC} 9 / 66$; it is a haploid prototrophic laboratory wild type. It was maintained by monthly subcultures on complete medium.

Media. The basal medium (minimal medium) had the following composition (Analar grade reagents): asparagine, I3 mM; ammonium tartrate, $3.3 \mathrm{mM} ; \mathrm{Na}_{2} \mathrm{HPO}_{4}$, Io $\mathrm{mM} ; \mathrm{KH}_{2} \mathrm{PO}_{4}$, IO $\mathrm{mM}$; $\mathrm{Na}_{2} \mathrm{SO}_{4}, 2 \mathrm{mM}$; thiamine hydrochloride, approximately $\mathrm{I} \times \mathrm{IO}^{-4} \%(\mathrm{w} / \mathrm{v})$. Hexoses were added to this medium in the concentrations indicated in the various experimental descriptions; normal hexoses were obtained from British Drug Houses Ltd., and 2-deoxy-D-glucose from the Sigma Chemical Company (London). A fully supplemented (complete) medium was prepared by adding glucose (to $100 \mathrm{~mm}$ ), Bacto Casamino acids $(0.07 \%, \mathrm{w} / \mathrm{v})$, Bacto yeast extract $(0.07 \%$, w/v), Bacto malt extract $(\mathrm{r} \cdot 0 \% \mathrm{w} / \mathrm{v})$, and hydrolysed nucleic acids (approximately $0.04 \%$, $\mathrm{w} / \mathrm{v})$ to the above. Media were solidified with Bacto agar $(\mathrm{I} \cdot 5 \% \mathrm{w} / \mathrm{v})$.

Preparation of oidial suspensions. In general monokaryotic strains of Coprinus produce asexual spores (oidia) continuously during the growth of the colony. These are formed by aerial hyphae and can be found in abundant numbers in fluid droplets in the aerial mycelium. Oidia were harvested from slope cultures of Coprinus lagopus BC $9 / 66$ on complete medium made up in 6 in. $\times \mathrm{I}$ in. Pyrex glass boiling tubes. Ten $\mathrm{ml}$. of distilled water were added to a tube after at least 5 days of incubation at $37^{\circ}$, and 
the oidia scraped into suspension with an inoculation needle or loop. After filtration through cotton-wool to remove mycelial debris the concentration of oidial suspensions was estimated with the aid of a haemacytometer slide. Viability of suspensions was determined by plating samples on complete medium agar and assessing what proportion of the visible oidia was able to grow into colonies. Where, in the sequel, results are given in terms of spore numbers these refer to the number of viable oidia, not to the total number of visible oidia.

Agitated suspensions. In the majority of experiments oidia were treated in suspension. The suspensions (Io ml.) were contained in I oz. McCartney bottles and continuously agitated by mounting on an angled turn-table within an incubator at $37^{\circ}$. At intervals I $\mathrm{ml}$. samples were removed, diluted and spread on to plates of complete medium. Plates were incubated at $37^{\circ}$ and colonies counted after about $48 \mathrm{hr}$.

Growth rates. The rate of extension growth was determined by measuring colony diameters at successive stages during incubation. Growth was initiated with $2 \mathrm{~mm}$. diam. disks cut with a punch from established (about 3 days old) complete medium cultures. Conditions of growth were standardized as far as possible; media were prepared and sterilized in one batch, $20 \mathrm{ml}$. quantities were used in plastic Petri dishes, and inoculated plates were incubated at $37^{\circ}$ together in a single incubator. Three replicates of each treatment were measured over two diameters at right angles.

Measurements of oxygen uptake. The rate of oxygen uptake by oidia in suspension was measured by using a conventional Warburg manometric apparatus at $37^{\circ}$. Warburg vessels contained in their main compartment, minimal medium constituents, oidia, and the required hexoses all in a total volume of $3.0 \mathrm{ml}$. All additions were adjusted in amount to give the required concentrations in this final volume. Oidial suspensions were adjusted to give a final concentration of $3 \times 10^{8}$ viable oidia $/ \mathrm{ml}$.

\section{RESULTS AND DISCUSSION}

\section{Viability of oidia}

The fungicidal action of 2-deoxy-D-glucose (deGlc) was first investigated by an agar overlay technique. Oidial viability was compared by incubating spores at $37^{\circ}$ on minimal medium containing $100 \mathrm{~mm}$-glucose and on minimal medium containing $100 \mathrm{~mm}-$ glucose $+5 \mathrm{~mm}$-deGlc. At $8 \mathrm{hr}$ intervals the plates were overlaid with complete medium, re-incubated until colonies appeared and these then counted. Survival was uniformly good $(75-100 \%)$ there being no significant difference between survival on the two types of medium even in those samples which had been exposed to deGlc for $48 \mathrm{hr}$. This technique was abandoned in favour of one using agitated oidial suspensions when it became clear that oidia would germinate and grow vigorously on minimal medium $+100 \mathrm{~mm}$-glucose $+5 \mathrm{~mm}$-deGlc. Medium overlaid on plates which had been incubated for more than about $48 \mathrm{hr}$ spread secondary oidia derived from established colonies, thus confusing the results.

The survival of oidia following treatment in suspensions containing various concentrations of glucose and deGlc is shown in Fig. I. Quantitative results were not reliable for most treatments of duration longer than about $25 \mathrm{hr}$, because of the appearance of colonies within the suspensions. Such colonies both trapped oidia and prevented accurate pipetting. Well-developed colonies were visible at the $25 \mathrm{hr}$ sample time in the controls and in those experiments containing $5 \mathrm{mM}-\mathrm{deGlc}+\mathrm{IO} \mathrm{mM}-\mathrm{glucose}$; 
at $32.5 \mathrm{hr}$ in $25 \mathrm{~mm}$-deGlc + ro mM-glucose; and small colonies were also seen in $50 \mathrm{~mm}$-deGlc + Io mM-glucose at $32 \cdot 5 \mathrm{hr}$. Very small colonies were also visible in the other experimental bottles at the end of the experiments. Despite this difficulty, however, the data clearly show that there was no very significant effect (if any) on oidial survival even at a 50: I molar ratio of deGlc:glucose. Furthermore, the appearance of colonies in the suspensions indicates that the coprinus was highly resistant to deGlc under these conditions.

Tests for oidial survival in liquid media containing fructose and deGlc were delayed until the extension-growth experiments were completed (see below). Survival in media containing $5 \mathrm{~mm}$ fructose $+0.5 \mathrm{~mm}$-deGlc was then examined, since growth was known

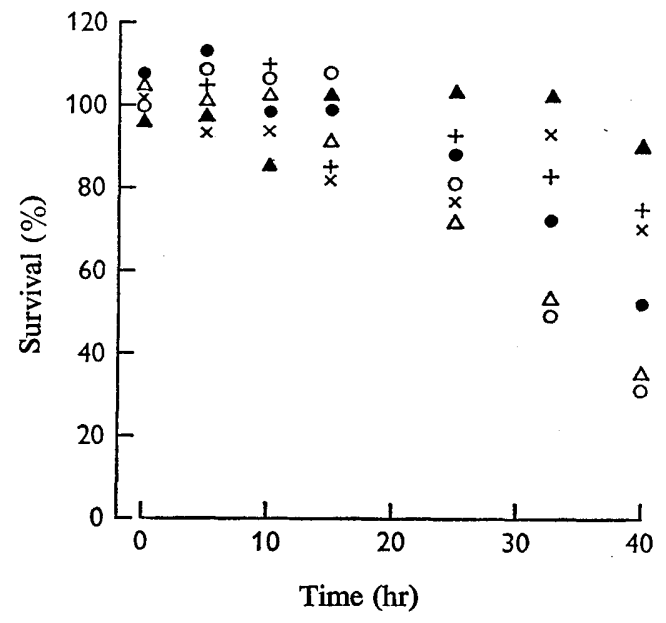

Fig. I

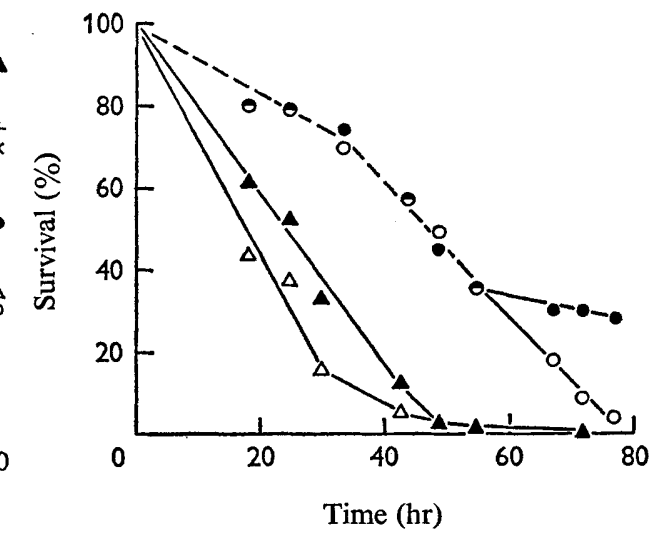

Fig. 2

Fig. I. Scatter diagram showing oidial viability following incubation in media containing different concentrations of glucose and deGlc. Viability at each point is expressed as $\%$ of the control value at zero time. Key: $O$, minimal medium containing Io mM-glucose (= control); $\triangle$, Io mM-glucose + 5 mM-deGlc; $\times$, Io mM-glucose $+25 \mathrm{~mm}$-deGlc; + , 10 mM-glucose + $50 \mathrm{~mm}$-deGlc;, $\mathrm{I} \cdot 0 \mathrm{~mm}$-glucose + $25 \mathrm{~mm}$-deGlc; $\Delta, \mathrm{I} \cdot 0 \mathrm{~mm}$-glucose $+50 \mathrm{~mm}$-deGlc.

Fig. 2. Curves showing oidial viability following incubation in four fungistatic media. Curve I (O) normal minimal medium containing 5 mm-fructose $+0.5 \mathrm{~mm}$-deGlc; curve $2(O)$ medium lacking all nitrogen and carbon sources (points showing no clear difference in viability in curves $I$ and 2 are plotted as half-closed circles); curve $3(\Delta)$ medium lacking nitrogen sources but containing $5 \mathrm{~mm}$-fructose $+0.5 \mathrm{~mm}$-deGlc; curve $4(\triangle)$ medium lacking nitrogen sources but containing $5 \mathrm{~mm}$-fructose.

not to occur on such media. The results given in Fig. 2 show the survival of oidia after incubation in several nutritionally deficient media. There was in general a distinct decrease in viability following incubation in fungistatic media. Though curve $I$ of Fig. 2 may suggest that the addition of deGlc to what was essentially the normal medium may have been the cause of the decreased viability, comparison with the other curves shows this was not the case. Any effect on viability in this system was a secondary consequence of the fungistatic action of deGlc and cannot be ascribed to a direct fungicidal action. It is concluded that, at least under the conditions tested, deGlc is not fungicidal towards the coprinus. 


\section{Inhibition of extension growth}

The rate of extension growth of the coprinus was profoundly affected by deGlc. As Fig. 3 and 4 show, the effective ratio of normal hexose to deGlc depended greatly on the identity of the normal hexose. Growth was decreased by about $50 \%$ on media containing equimolar amounts of glucose and deGlc; a similar degree of inhibition was obtained with fructose + deGlc, but with these compounds in a molar ratio of IOO: I.

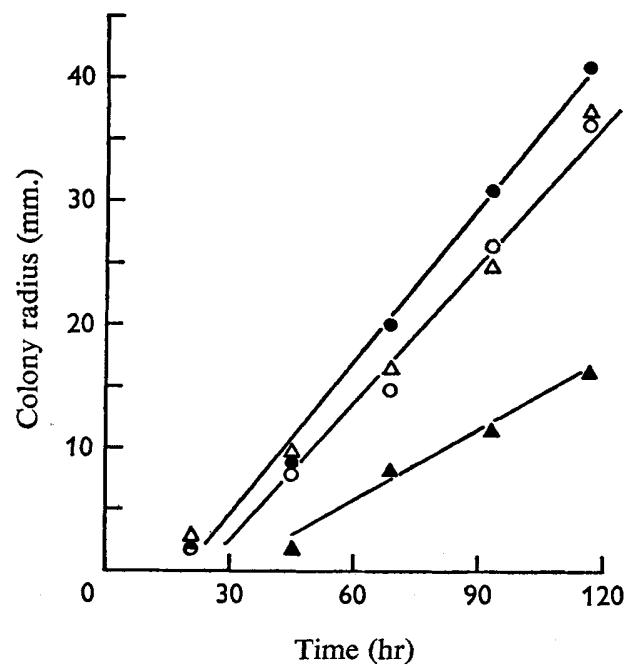

Fig. 3

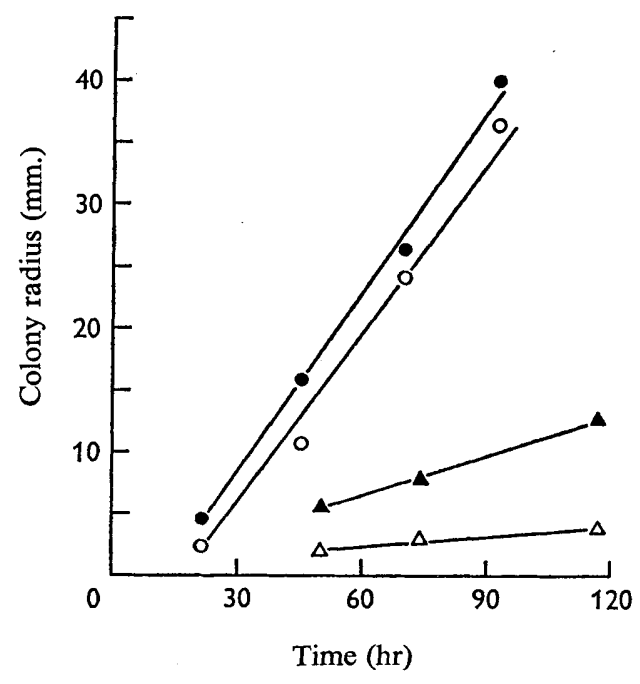

Fig. 4

Fig. 3. Growth rates of colonies on solid media containing: 5 mM-glucose $(\bullet) ; 2.5 \mathrm{~mm}$ glucose $(O) ; 5$ mM-glucose $+5 \mathrm{~mm}$-deGlc $(\Delta) ; 5$ mM-glucose $+0.5 \mathrm{~mm}$-deGlc $(\triangle)$.

Fig. 4. Growth rates of colonies on solid media containing: 10 mM-fructose $(0)$; $5 \mathrm{~mm}$ fructose $(O)$; 5 mM-fructose + $0.05 \mathrm{~mm}$-deGlc ( $\Delta$ ); 5 mM-fructose + 0.25 mM-deGlc $(\triangle)$.

No growth at all was observed within $\mathrm{I} 20 \mathrm{hr}$ on media containing deGlc alone; concentrations up to $10 \mathrm{~mm}$ were tested. Similarly, no growth was obtained on media containing equimolar ( $5 \mathrm{mM}$ ) amounts of fructose and deGlc; $5 \mathrm{~mm}$-fructose $+2.5 \mathrm{~mm}$ deGlc; or $5 \mathrm{~mm}$-fructose $+0.5 \mathrm{~mm}$-deGlc. This fungistatic influence of deGlc towards the coprinus was annulled by adding extra amounts of glucose or fructose; 5 to 10 times as much fructose was required as compared with glucose. This annulment of inhibition could be effected up to the maximum time that inocula were maintained on fungistatic media ( $120 \mathrm{hr}$ ). Similarly, inocula removed from fungistatic media to normal medium; even after $\mathbf{I} 20 \mathrm{hr}$, grew immediately with normal vigour.

These experiments made it clear that deGlc was not utilized by the coprinus when it was the sole available hexose, and that its fungistatic effects could be completely annulled. The experiments revealed an interesting pattern of sensitivity to deGlc. It appears from the curves in Fig. 3 and 4 that, judging from effective concentrations, the coprinus was at least 100 times more resistant to the effects of deGlc when glucose was present than when fructose was present. Since it is known from oidial survival experiments that colonies appear within $40 \mathrm{hr}$ in media containing deGlc:glucose in 
a 50: I ratio, it is highly likely that this sensitivity differential is very much more pronounced than that indicated here. The problem of this differential sensitivity will be returned to later.

\section{Oxygen uptake}

Although deGlc alone did not support growth, oxygen uptake did proceed (Fig. 5); though only at a rate about $25 \%$ of that observed on the same concentration of glucose. The same comparison can be made between growth and $\mathrm{O}_{2}$ uptake on $5 \mathrm{~mm}$ fructose $+5 \mathrm{~mm}$-deGlc. In general the inhibitory effect of deGlc on $\mathrm{O}_{2}$ uptake followed fairly closely its effect on growth; the differences were largely a matter of degree. This was also a feature of previous studies; it would appear that the inhibition of a single metabolic process was less dramatic than the cumulative inhibition of the entire

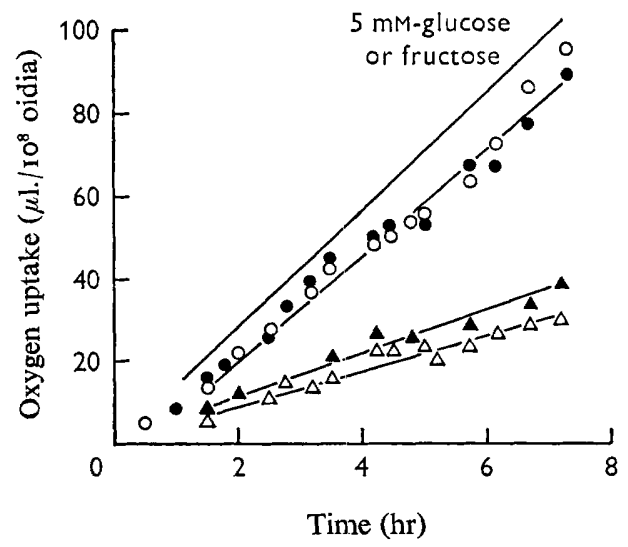

Fig. 5

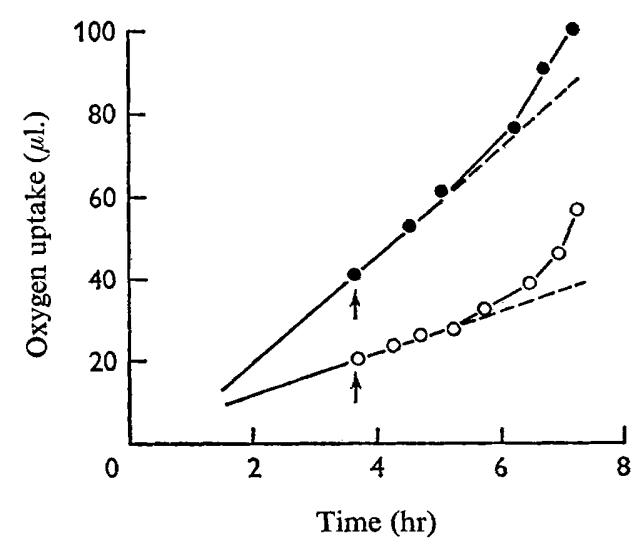

Fig. 6

Fig. 5. Oxygen consumption by spores suspended in liquid media containing: $5 \mathrm{~mm}$-deGlc $(\triangle)$; 5 mM-glucose + 5 mM-deGlc (O) ; 5 mM-fructose + 5 mM-deGlc ( $\Delta$ ); 5 mM-fructose + 0.05 mMdeGlc (O).

Fig. 6. Annulment of the inhibitory effects of deGlc. At the times indicated by the arrows, glucose to a final concentration of $15 \mathrm{~mm}$ was tipped from the side-arm into the main compartment of Warburg flasks. Up to that point the spores had been suspended in media containing: 5 mM-glucose + 5 mm-deGlc (O); 5 mM-fructose + 5 mM-deGlc (O).

metabolic complex. Addition of more normal substrate to vessels containing an inhibitory concentration of deGlc annulled that inhibition (Fig. 6); glucose was at least 5 to ro times more effective than fructose.

The graphs in Fig. 5 show that the differential sensitivity previously referred to applied as much to $\mathrm{O}_{2}$ uptake as to extension growth. The rate of $\mathrm{O}_{2}$ uptake measured in experiments involving $5 \mathrm{~mm}$ glucose $+5 \mathrm{mM}$ deGlc was very similar to the rate measured in experiments with $5 \mathrm{~mm}$ fructose $+0.05 \mathrm{~mm}$ deGlc; there was at least a Ioo-fold difference in sensitivity.

It is clear that both growth and $\mathrm{O}_{2}$ uptake of the coprinus were much affected by deGlc, and that the inhibition was readily annulled. There was an extreme difference in sensitivity depending on the identity of the normal hexose. Differential sensitivity of this sort has been noted before, but not on so great a scale as observed here. Barban \& Schulze (I96I) quoted, for human cells, a 5 to Io times greater sensitivity 
with fructose as compared with glucose; from the data of Heredia et al. (1964) it appears that Saccharomyces cerevisiae was about twice as sensitive to the growth inhibitory effects of deGlc in the presence of fructose. In both of these cases the effects in experiments with mannose and deGlc were virtually identical to those observed with glucose and deGlc. In these two studies, the most closely related in scope to the present one, no growth occurred on equimolar glucose + deGlc; growth of the coprinus was only $50 \%$ inhibited under such conditions. The sensitivity of the coprinus on fructose media was closely similar to the general sensitivity pattern of normal yeast and human cells. On the other hand the coprinus sensitivity on media containing glucose paralleled the sensitivity of resistant sub-lines of HeLa cells (Barban, 1962) and resistant mutants of yeast (Heredia \& Sols, 1964). Yet these extremes in the expression of sensitivity have been observed in the same strain of Coprinus lagopus. There are many steps in sugar metabolism capable of providing a reasonable explanation for this sensitivity differential. Among these are, for example, competitive inhibition of sugar uptake favouring the aldohexose at the expense of the ketohexose, or a much greater inhibition of fructose phosphorylation than glucose phosphorylation, possibly due to a lesser affinity of hexokinase for fructose. Preliminary experiments suggest that the overall sensitivity difference may well be the cumulative result of several different individual effects.

My thanks are due to Dr D. H. Morgan of the John Innes Institute for supplying me with the Coprinus lagopus strain $\mathrm{BC} 9 / 66$ used in this work.

\section{REFERENCES}

BARban, S. (1962). Studies on the mechanism of resistance to 2-deoxy-D-glucose in mammalian cell cultures. J. biol. Chem. 237, 291.

Barban, S. \& Schulze, H. O. (1961). The effects of 2-deoxyglucose on the growth and metabolism of cultured human cells. J. biol. Chem. 236, I887.

Heredia, C. F. \& Sols, A. (1964). Metabolic studies with 2-deoxyhexoses. II. Resistance to 2-deoxyglucose in a yeast mutant. Biochim. biophys. Acta 86, 224.

Heredia, C. F., De la Fuente, G. \& Sols, A. (1964). Metabolic studies with 2-deoxyhexoses. I. Mechanisms of inhibition of growth and fermentation in bakers yeast. Biochim. biophys. Acta 86, 216.

MegNet, R. (1965). Screening of auxotrophic mutants of Schizosaccharomyces pombe with 2-deoxyglucose. Mutation Res. 2, 328.

Sols, A., Heredia, C. F. \& Rutz-Amil, M. (1960). 2-Deoxyglucose as metabolic substrate and inhibitor of glycolysis in Fungi. Biochem. biophys. Res. Commun. 2, 126.

Webi, J. L. (1966). Enzyme and Metabolic Inhibitors, vol. 2, p. 386. London: Academic Press.

Woodward, G. E., Cramer, F. B. \& Hudson, M. T. (1953). Carbohydrate analogs as antagonists of glucose in carbohydrate metabolism of yeast. J. Franklin Inst. 256, 577. 